\title{
Microscopic approach to polaritons
}

\author{
Skettrup, Torben
}

Published in:

Physical Review B

Link to article, DOI:

10.1103/PhysRevB.24.884

Publication date:

1981

Document Version

Publisher's PDF, also known as Version of record

Link back to DTU Orbit

Citation (APA):

Skettrup, T. (1981). Microscopic approach to polaritons. Physical Review B, 24(2), 884-891. https://doi.org/10.1103/PhysRevB.24.884

\section{General rights}

Copyright and moral rights for the publications made accessible in the public portal are retained by the authors and/or other copyright owners and it is a condition of accessing publications that users recognise and abide by the legal requirements associated with these rights.

- Users may download and print one copy of any publication from the public portal for the purpose of private study or research.

- You may not further distribute the material or use it for any profit-making activity or commercial gain

- You may freely distribute the URL identifying the publication in the public portal

If you believe that this document breaches copyright please contact us providing details, and we will remove access to the work immediately and investigate your claim. 


\title{
Microscopic approach to polaritons
}

\author{
T. Skettrup \\ Physics Laboratory III, Technical University of Denmark, DK-2800 Lyngby, Denmark
}

(Received 6 October 1980)

\begin{abstract}
The interaction between excitons and light has been investigated in detail. The perturbational approach turns out to be invalid. However, an exact solution can be obtained directly from the Schrödinger equation for a fixed light field. This solution corresponds to a nonlinear optical response contrary to experimental experience. In order to remove this absurdity the semiclassical approach must be abandoned and the electromagnetic field quantized. A simple microscopic polariton model is then derived. From this the wave function for the interacting exciton-photon complex is obtained. It is discussed whether the magnitude of the exciton-photon interaction energy has any influence on the exciton binding. The concept of polariton fields is introduced (i.e., the electric and polarization field per polariton), and it is seen that close to resonance the electric field per polariton is essentially zero while the polarization field per polariton is essentially the excitonic dipole moment per unit volume. From the wave function of the polariton it is seen that in a very broad spectral range around the resonance the polariton is essentially an exciton that travels through the crystal with the speed of light of the crystal. The introduction of damping smears out the excitonic spectra. The wave function of the polariton, however, turns out to be very independent of damping up to large damping values. Finally, this simplified microscopic polariton model is compared with the exact solutions obtained for the macroscopic polariton model by Hopfield. It is seen that standing photon and exciton waves must be included in an exact microscopic polariton model. However, it is concluded that for practical purposes, only the propagating waves are of importance and the simple microscopic polariton wave function derived in the present work is then sufficient.
\end{abstract}

\section{INTRODUCTION}

The polariton is usually considered as the elementary quantum of the coupled propagating electromagnetic field and polarization field of the crystal. The properties of the polaritons have, therefore, been derived from the macroscopic equations of motion for the fields by means of quantization. However, the polaritons possess an internal structure determined by the properties of the quanta of the pure polarization (i.e., excitons, phonons, etc.). The purpose of the present work is to derive these internal properties of the polaritons (i.e., the wave function) in the case of interaction between excitons and light. Hence, it is necessary to apply a microscopic approach to the interaction problem instead of the usual macroscopic approach employed by Hopfield. ${ }^{1}$

Section II serves as an introduction to this microscopic interaction. The problem is introduced in terms of the usual perturbational approach. However, this method turns out to be invalid, and in Sec. III it is shown that the problem of an exciton interacting with a fixed electromagnetic field can be solved exactly in a certain approximation. From this semiclassical solution it is seen that the solution appears meaningless unless the exciton is interacting not with field, but with the quantum of the field (the photon). A simple microscopic approach to the exciton-photon interaction problem is then given in Sec. IV. The wave func- tion for the interacting exciton-photon complex (the polariton) is found. The question of whether the exciton can bind together when the excitonphoton interaction energy exceeds the binding energy is discussed in terms of this wave function. The results obtained from this simplified microscopic polariton model is then compared with the results from the exact macroscopic model of Hopfield in Sec. V.

\section{PERTURBATIONAL APPROACH}

The perturbational approach to the treatment of the interaction between light and excitons is well known (see, for example, Ref. 2) and will not be described here in any detail. We shall only, as an introduction to the following, state the results and discuss why they, in principle, are wrong.

The interaction term in the Hamiltonian is given by

$$
V=-e \sum_{i} \overrightarrow{\mathrm{E}}_{0} \cdot \overrightarrow{\mathrm{r}}_{i} \cos \left(\overrightarrow{\mathrm{k}} \cdot \overrightarrow{\mathrm{r}}_{i}-\omega t\right)
$$

where $E_{0}$ is the amplitude of the electric field of the light wave with wave vector $\overrightarrow{\mathrm{k}}$ and angular frequency $\omega$, and $\vec{r}_{i}$ is the coordinate of the $i$ th electron. The perturbed wave function is

$$
\Psi_{p}=a_{1}(t) \Psi_{G}+a_{2}(t) \Psi_{\mathrm{ex}},
$$


where

$$
\Psi_{G}=\Phi_{0} e^{-i \omega_{0} t}
$$

and

$$
\Psi_{\mathrm{ex}}=\Phi_{1} e^{-i \omega_{1} t} .
$$

$\Phi_{0}$ and $\Phi_{1}$ are wave functions for the crystal ground state and $n=1$ exciton state, respectively. Excited exciton states are omitted in (2) since their interaction with light decreases with $n^{3}$. The resonance frequency of the exciton $(n=1)$ state is

$$
\omega_{\mathrm{ex}}=\omega_{1}-\omega_{0} \text {. }
$$

Inserting (2) into the time-dependent Schrödinger equation we obtain

$$
\begin{aligned}
& i \hbar \frac{d a_{1}}{d t}=V_{12} a_{2}, \\
& i \hbar \frac{d a_{2}}{d t}=V_{21} a_{1},
\end{aligned}
$$

where

$$
\begin{aligned}
V_{21}=V_{12}^{*}=- & \frac{e \overrightarrow{\mathrm{E}}_{0} \cdot\langle\overrightarrow{\mathrm{r}}\rangle \phi}{2} \\
& \times\left(e^{i\left(\overrightarrow{\mathrm{k}} \cdot \overrightarrow{\mathrm{R}}+\omega_{\mathrm{ex}^{t}-\omega t}\right)}+e^{-i\left(\overrightarrow{\mathrm{k}} \cdot \overrightarrow{\mathrm{R}}-\omega_{\mathrm{ex}} t-\omega t\right)}\right)
\end{aligned}
$$

Here $\langle\overrightarrow{\mathbf{r}}\rangle$ is the $r$-matrix element between the Wannier functions from the valence and conduction bands ${ }^{2}$ and

$$
\phi=\int F^{*}\left(\overrightarrow{\mathrm{R}}_{e}, \overrightarrow{\mathrm{R}}_{e}\right) d^{3} R_{e}=\left(\frac{N \Omega}{\pi a_{B}^{3}}\right)^{1 / 2} \delta \overrightarrow{\mathrm{K}}_{,} \overrightarrow{\mathrm{k}},
$$

where $F\left(\overrightarrow{\mathrm{R}}_{e}, \overrightarrow{\mathrm{R}}_{h}\right)$ is the envelope function for the exciton, $N$ is the number of unit cells, $\Omega$ the unit cell volume, $a_{B}$ the exciton radius, $\vec{K}$ and $\vec{R}$ [in (8)] are wave vector and coordinate of center of gravity.

Now, in the perturbational approach, an approximate expression for (2) is obtained by assuming $a_{1} \simeq 1$ and $a_{2} \ll a_{1}$. Using the initial conditions

$$
a_{1}(0)=1, \quad a_{2}(0)=0
$$

and neglecting the $\left(\omega_{\mathrm{ex}}+\omega\right)$ term in (8), one finds as the approximate solutions of (6) and (7)

$a_{1}(t) \simeq 1, \quad a_{2}(t) \simeq \frac{e \overrightarrow{\mathbf{E}}_{0} \cdot\langle\overrightarrow{\mathrm{r}}\rangle \phi}{2} \frac{e^{i \overrightarrow{\mathrm{k}} \cdot \overrightarrow{\mathrm{R}}}\left(e^{i\left(\omega_{\mathrm{ex}}-\omega\right) t}-1\right)}{\hbar\left(\omega_{\mathrm{ex}}-\omega\right)}$.

With (11), the induced dipole moment is

$$
\langle\vec{\mu}\rangle=\left\langle\psi_{p}\left|\sum_{i} e \overrightarrow{\mathrm{r}}_{i}\right| \psi_{p}\right\rangle \simeq \overrightarrow{\mathrm{E}}_{0} \frac{e^{2}\langle r\rangle^{2} \phi^{2}}{3 \hbar\left(\omega_{\mathrm{ex}}-\omega\right)} \cos (\overrightarrow{\mathrm{k}} \cdot \overrightarrow{\mathrm{R}}-\omega t),
$$

neglecting the transient due to the initial conditions. The macroscopic polarization $P_{\mathrm{ex}}$ is the average dipole moment per unit volume. Hence in the volume $V$,

$$
\overrightarrow{\mathrm{P}}_{\mathrm{ex}}=\frac{\langle\vec{\mu}\rangle}{V}=\frac{(N / V) e^{2} f_{\mathrm{ex}}}{m\left(\omega_{\mathrm{ex}}-\omega\right) 2 \omega_{\mathrm{ex}}} \overrightarrow{\mathrm{E}}_{0} \cos (\overrightarrow{\mathrm{k}} \cdot \overrightarrow{\mathrm{R}}-\omega t),
$$

where

$$
f_{\mathrm{ex}}=\frac{2 m \omega_{\mathrm{ex}}}{3 \hbar} \frac{\langle r\rangle^{2} \phi^{2}}{N}
$$

is the oscillator strength per unit cell. From (13), then, the well known dielectric constant is found,

$$
\epsilon=\epsilon^{\prime}+\frac{F}{\omega_{\mathrm{ex}}^{2}-\omega^{2}},
$$

where $\epsilon^{\prime}$ is the background dielectric constant, and where $\omega_{\mathrm{ex}}^{2}-\omega^{2} \simeq 2 \omega_{\mathrm{ex}}\left(\omega_{\mathrm{ex}}-\omega\right)$, while

$$
F=\frac{(N / V) e^{2} f_{\mathrm{ex}}}{m \epsilon_{0}}
$$

where $\epsilon_{0}$ is the vacuum permittivity. From (11) we also obtain the transition probability per unit time,

$$
W=\frac{\left|a_{2}(t)\right|^{2}}{t}-2 \pi \frac{\left(\mu_{0} E_{0}\right)^{2} N}{4 \hbar^{2}} \delta\left(\omega_{\mathrm{ex}}-\omega\right),
$$

where

$$
\mu_{0}=\frac{e|\langle r\rangle|}{\sqrt{3}}\left(\frac{\Omega}{\pi a_{B}^{3}}\right)^{1 / 2} .
$$

Hence, (13) and (17) are the results of the perturbational approach. It is evident that (17) is incorrect. Owing to the conservation of $k$ [see Eq. (9)] there are no final states to sum over to remove the $\delta$ function. Furthermore, according to (17) the transition probability should be dependent on the size of the crystal, which is wrong. It is surprising, therefore, that (13) is correct. However, no estimates of the magnitude of $E_{0}$ and $\omega_{\text {ex }}-\omega$ are given to show when (13) can be applied. It is common to state that $E_{0}$ of light can be made arbitrarily small so that the perturbational approach can be used. This is not true, however, due to the corpuscular nature of light. The photon-exciton interaction energy ( $\mu E_{0}$, see below) is actually so large for most materials that the perturbational approach is not valid.

\section{III. "EXACT" SOLUTION}

Fortunately, (6) and (7) can be solved exactly in the approximation where the $\omega_{\text {ex }}+\omega$ terms are neglected (as was also done in Sec. I). Equations (6) and (7) can in this approximation be written as

$$
\begin{aligned}
& \dot{a}_{2}=A e^{i\left(\omega_{\mathrm{ex}}-\omega\right) t} a_{1}, \\
& \dot{a}_{1}=-A^{*} e^{-i\left(\omega_{\mathrm{ex}}-\omega\right) t} a_{2},
\end{aligned}
$$


where

$$
A=-\frac{\mu E_{0}}{2 i \hbar} e^{i \overrightarrow{\mathrm{k}} \cdot \overrightarrow{\mathrm{R}}}
$$

and

$$
\mu=\mu_{0} \sqrt{N} .
$$

It is seen that $a_{1}=\exp \left(i p_{1,2} t\right)$ is a solution to (19) and $(20)$ when

$$
p_{1,2}=-\frac{1}{2}\left(\omega_{\mathrm{ex}}-\omega\right) \pm \frac{1}{2} p,
$$

where

$$
p=p_{2}-p_{1}=\left[\left(\omega_{\mathrm{ex}}-\omega\right)^{2}+\omega_{c}^{2}\right]^{1 / 2}
$$

with

$$
\hbar \omega_{c}=2 \hbar|A|=\mu E_{0} .
$$

$\mu E_{0}$ is the interaction energy between the applied field and the induced dipole moment. A solution is, then,

$$
\begin{aligned}
& a_{1}(t)=\left(\frac{p_{2}}{p}\right)^{1 / 2} e^{i p_{1} t}, \\
& a_{2}(t)=-\frac{i p_{1}}{A^{*}}\left(\frac{p_{2}}{p}\right)^{1 / 2} e^{i\left(p_{1}+\omega_{\mathrm{ex}}-\omega\right) t},
\end{aligned}
$$

since $\left|a_{1}\right|^{2}+\left|a_{2}\right|^{2}=1$ and $A A^{*}=-p_{1} p_{2}$. From (2) and (12) we obtain using (26) and (27)

$$
\overrightarrow{\mathrm{P}}_{\mathrm{ex}}=\frac{\langle\vec{\mu}\rangle}{V}=\frac{(N / V) \mu_{0}^{2} \overrightarrow{\mathrm{E}}_{0} \cos (k R-\omega t)}{\hbar\left[\left(\omega_{\mathrm{ex}}-\omega\right)^{2}+\omega_{c}^{2}\right]^{1 / 2}} .
$$

This is then the exact polarization response due to the fixed light field $E_{0}$. Hence (13) is only valid for

$$
\left|\omega_{\mathrm{ex}}-\omega\right| \gg \omega_{c} .
$$

It is seen that $\omega_{c}$ is dependent on the field $E_{0}$. $\omega_{c}$ must, therefore, be a varying quantity. The solution (28) corresponds, then, to a nonlinear dielectric response. This appears to be rather meaningless and in contradiction with experimental experience. The problem can only be solved by giving up the semiclassical description and instead using directly the fact that the interaction between light and matter takes place via exchange of photons. The solution (28) can then be applied if $E_{0}$ is interpreted as the "electric field per photon." This field is given in (45) and discussed there in more detail. Introducing $E_{0}$ from (45) we obtain

$$
\begin{aligned}
\omega_{c} & =\frac{\mu E_{0}}{\hbar}=\left(\frac{\mu_{0}^{2} N \hbar \omega}{2 \hbar^{2} \epsilon_{0} \epsilon^{\prime} V}\right)^{1 / 2} \\
& =\left(\frac{1}{2} \omega_{\mathrm{LT}} \omega\right)^{1 / 2} \simeq\left(\frac{1}{2} \omega_{\mathrm{LT}} \omega_{\mathrm{ex}}\right)^{1 / 2}=\left(\frac{F}{4 \epsilon^{\prime}}\right)^{1 / 2},
\end{aligned}
$$

where

$$
\omega_{\mathrm{LT}}=\frac{F}{2 \epsilon^{\prime} \omega_{\mathrm{ex}}}=\frac{(N / V) \mu_{0}^{2}}{\hbar \epsilon_{0} \epsilon^{\prime}}
$$

is the splitting between transverse and longitudinal resonance frequencies of the excitons [see Eq. (15)].

The polarization (28) introduced by a photon with energy $\hbar \omega$ is then

$$
P_{\mathrm{ex}}=P_{1} \frac{\omega_{c}}{\left[\left(\omega_{\mathrm{ex}}-\omega\right)^{2}+\omega_{c}^{2}\right]^{1 / 2}} \cos (k R-\omega t),
$$

where

$$
P_{1}=\frac{\mu}{V}
$$

is the "polarization per (pure) exciton."

An electromagnetic field can only propagate through the solid if the macroscopic wave equations for electric field and excitonic polarization are simultaneously obeyed. This is only the case if

$$
k^{2}=\frac{\omega^{2}}{c^{2}} \epsilon(\omega)
$$

and

$$
P_{\mathrm{ex}} / E=\epsilon_{0}\left(\epsilon(\omega)-\epsilon^{\prime}\right),
$$

where

$$
\epsilon(\omega)=\epsilon^{\prime}+\frac{F}{\omega_{\mathrm{ex}}^{2}-\omega^{2}} \simeq \epsilon^{\prime}+\frac{F}{2 \omega_{\mathrm{ex}}\left(\omega_{\mathrm{ex}}-\omega\right)} .
$$

Equation (34) determines the dispersion relation between $\omega$ and $k$ for propagating solutions. From (35) we find by introducing (32) and using (36) that the electric field of the propagating electromagnetic mode due to the exciton polarization (32) is given by

$$
E=E_{1} \frac{\omega_{\mathrm{ex}}-\omega}{\left[\left(\omega_{\mathrm{ex}}-\omega\right)^{2}+\omega_{c}^{2}\right]^{1 / 2}} \cos (k R-\omega t),
$$

where $E_{1}$ is the "electric field per (pure) photon" given by (45) as seen from (31). Hence, the incoming photon with energy $\hbar \omega$ gives rise to a propagating polarization of magnitude (37). The total energy of the combined waves is

$$
W=2 \frac{1}{2} \epsilon_{0} \epsilon^{\prime} E^{2} V+2 \frac{1}{2 \epsilon_{0} F}\left(\frac{d P}{d t}\right)^{2} V=\hbar \omega,
$$

as seen from (32) and (37). Hence, this combined wave system itself represents a fundamental quantum $\hbar \omega$ propagating through the crystal with $\omega$ and $k$ obeying (34). The macroscopic coupled electromagnetic and polarization field is built up of these quanta, and (32) and (37) can be interpreted as the "fields per quantum." It is seen, therefore, that this quantum is the same as the polariton introduced by Hopfield ${ }^{1}$ by means of quantiza- 
tion of the coupled macroscopic equations of motion for electric and polarization fields. Here it is obtained from a microscopic approach. The electric field was, however, still treated via the macroscopic equations, since it was not included in the Hamiltonian. This implies, for example, that (26) and (27) will not yield the correct exciton (polariton) wave function when inserted into (2) because the change in the electric field due to the interaction was not taken into account in the derivation of (26) and (27), and both the $\omega-k$ relationship and the ratio $P / E$ are different for the solutions obtained from (26) and (27) than for those found in (34) and (35).

\section{SIMPLE POLARITON MODEL}

In order to obtain the correct wave function for the exciton interacting with light it is, therefore, necessary to include the electric field directly into the Hamiltonian,

$$
\left(\hat{H}_{\mathrm{ex}}+\hat{H}_{\mathrm{ph}}+\hat{H}_{i}\right) \psi=i \hbar \frac{\partial \psi}{\partial t}
$$

where $\hat{H}_{\text {ex }}, \hat{H}_{\mathrm{ph}}$, and $\hat{H}_{i}$ are pure exciton, pure photon, and exciton-photon interaction Hamiltonians, respectively. The problem specified by (39) was solved exactly by Hopfield ${ }^{1}$ from a macroscopic approach where the macroscopic electromagnetic and polarization fields were quantized. Here we are interested in obtaining the microscopic wave function for the excitonic polariton, and only the photon Hamiltonian is then derived from the macroscopic fields,

$$
\hat{H}_{\mathrm{ph}}=\hbar \omega_{p} \sum_{k}\left(a_{k}^{\dagger} a_{k}+\frac{1}{2}\right),
$$

where $a_{k}$ is a photon annihilation operator, and

$$
\omega_{p}=k \frac{c}{\sqrt{\epsilon^{\prime}}}
$$

is the frequency corresponding to the noninteracting photon with wave vector $\vec{k}$. The operator for the macroscopic vector potential is (when quantization is performed in a homogeneous material with dielectric constant $\epsilon^{\prime}$ and volume V)

$$
\hat{A}=A_{1} \sum_{k}\left(a_{k} e^{i \overrightarrow{\mathrm{k}} \cdot \overrightarrow{\mathrm{r}}}+a_{k}^{\dagger} e^{-i \overrightarrow{\mathrm{k}} \cdot \overrightarrow{\mathrm{r}}}\right)
$$

when only one polarization is considered. Here

$$
A_{1}=\left(\frac{\hbar}{2 \epsilon_{0} \epsilon^{\prime} \omega_{p} V}\right)^{1 / 2} \text {. }
$$

is

$$
\hat{E}=i E_{1} \sum_{k}\left(a_{k} e^{i \overrightarrow{\mathrm{k}} \cdot \overrightarrow{\mathrm{r}}}-a_{k}^{\dagger} e^{-i \overrightarrow{\mathrm{k}} \cdot \overrightarrow{\mathrm{r}}}\right)
$$

with

$$
E_{1}=\left(\frac{\hbar \omega_{p}}{2 \epsilon_{0} \epsilon^{\prime} V}\right)^{1 / 2}
$$

$E_{1}$ can be considered as the "electric field per pure photon state." Equation (45) was applied in (30) to derive the magnitude of $\omega_{c}$.

Owing to the quantization of the electric field the interaction Hamiltonian (1) is changed into

$$
\hat{H}_{i}=-i e E_{1} \sum_{i, k} \overrightarrow{1}_{E} \cdot \overrightarrow{\mathrm{r}}_{i}\left(a_{k} e^{i \overrightarrow{\mathrm{k}} \cdot \overrightarrow{\mathrm{r}}}-a_{k}^{\dagger} e^{-\vec{i} \cdot \overrightarrow{\mathrm{k}}}\right)
$$

where $\overrightarrow{1}_{E}$ is a unit vector along the $E$ field. With (46) and (40) introduced into (39) a solution is the superposition

$$
\psi=a_{1}(t) \Psi_{0} \Phi_{1} e^{-i \omega_{p^{t}}}+a_{2}(t) \Psi_{1} \Phi_{0} e^{-i \omega_{\mathrm{ex}} t},
$$

where $\Psi_{0}$ and $\Phi_{0}$ are the crystal and vacuum ground states, respectively, while $\Psi_{1}$ and $\Phi_{1}$ are the exciton state $(n=1)$ and the one-photon state with wave vector $\vec{k}$. Inserting (47) into (39), we obtain in the usual approximation where the nonresonant $\omega_{\mathrm{ex}}+\omega_{p}$ terms are neglected

$$
\begin{aligned}
& \frac{d a_{1}}{d t}=\omega_{c} a_{2} e^{-i\left(\omega_{\mathrm{ex}}-\omega_{p}\right) t}, \\
& \frac{d a_{2}}{d t}=-\omega_{c} a_{1} e^{i\left(\omega_{\mathrm{ex}}-\omega_{p}\right) t}
\end{aligned}
$$

when (8), (9), and (30) are used. It is seen that $a_{1}=\exp \left(-i s_{1,2} t\right)$ is a solution to (48) and (49) when

$$
s_{1,2}=\frac{1}{2}\left(\omega_{\mathrm{ex}}-\omega_{p}\right) \pm \frac{1}{2}\left[\left(\omega_{\mathrm{ex}}-\omega_{p}\right)^{2}+4 \omega_{c}^{2}\right]^{1 / 2} .
$$

Considering either $s_{1}$ or $s_{2}$ we then find from the normalization condition

$$
\begin{aligned}
& a_{1}(t)=\left(\frac{\omega_{c}^{2}}{s_{1,2}^{2}+\omega_{c}^{2}}\right)^{1 / 2} e^{-i s_{1,2} t}, \\
& a_{2}(t)=-i \frac{s_{1,2}}{\omega_{c}}\left(\frac{\omega_{c}^{2}}{s_{1,2}^{2}+\omega_{c}^{2}}\right)^{1 / 2} e^{-i\left(s_{1,2}-\omega_{\mathrm{ex}}+\omega_{p}\right) t} .
\end{aligned}
$$

Introducing (51) and (52) into (47), the common time factor $\left(s_{1,2}+\omega_{p}\right) t$ is found. Hence, the mixed state $(47)$ is a stationary state, and the eigenenergies of the mixed state (47) (the excitonic polariton) are given by

$$
\begin{aligned}
\hbar \omega_{1,2} & =\hbar\left(s_{1,2}+\omega_{p}\right) \\
& =\frac{1}{2} \hbar\left\{\omega_{\mathrm{ex}}+\omega_{p} \pm\left[\left(\omega_{\mathrm{ex}}-\omega_{p}\right)^{2}+4 \omega_{c}^{2}\right]^{1 / 2}\right\} .
\end{aligned}
$$

From (50) and (53) the following relationships are 
obtained:

$\left(\omega_{1,2}-\omega_{p}\right)\left(\omega_{\mathrm{ex}}-\omega_{1,2}\right)=s_{1,2}\left(\omega_{\mathrm{ex}}-\omega_{1,2}\right)=-\omega_{c}^{2}$.

Introducing (54) into (51) and (52), the wave function for the excitonic polariton can be written as

$$
\begin{aligned}
\psi= & \left(\frac{\omega_{\mathrm{ex}}-\omega}{\left[\left(\omega_{\mathrm{ex}}-\omega\right)^{2}+\omega_{c}^{2}\right]^{1 / 2}} \Psi_{0} \Phi_{1}\right. \\
& \left.+i \frac{\omega_{c}}{\left[\left(\omega_{\mathrm{ex}}-\omega\right)^{2}+\omega_{c}^{2}\right]^{1 / 2}} \Psi_{1} \Phi_{0}\right) e^{-i \omega t},
\end{aligned}
$$

where indices 1 and 2 are omitted. Hence (55) is a solution of (39) with the eigenenergies (53). Equation (55) is the wave function for the excitonic polariton (or the dipole-active exciton interacting with light) in the approximation where nonresonant terms (such as $\omega_{\mathrm{ex}}+\omega_{p}$ ) are neglected. Furthermore, the interaction of the excitons with the background dielectric material was neglected in (39). This interaction has, essentially, the effect that $\omega_{\mathrm{ex}}$ is shifted by the amount of $\omega_{\mathrm{LT}}$ [given by (31)] towards higher frequencies. As shown later, the incorporation of above points to obtain the exact solution results in an unnecessarily complicated wave function. In the present simplified polariton model this is avoided.

With the wave function (55) for the interacting exciton-photon complex derived, several important questions can be answered. For many crystals, for example, $\hbar_{\omega_{c}}>E_{B}$, i.e., the excitonphoton interaction energy is greater than the exciton binding energy $E_{B}$. When this is the case, one should expect that the exciton-photon interaction is so strong that the exciton can no longer bind together. This is, however, not the case as shown above. The pure exciton energy $\hbar \omega_{\text {ex }}=E_{\text {gap }}-E_{B}$ is changed to $\hbar \omega_{1}$ (or $\hbar \omega_{2}$ ) given by (53) by the interaction with photons. This new eigenenergy of the interacting exciton (the polariton) is just composed of independent contributions from the exciton $\left(\omega_{\text {ex }}\right)$, from the photon $\left(\omega_{p}\right)$, and from the interaction $\left(\omega_{c}\right)$. The exciton binding is not destroyed by the exciton-photon interaction. The binding energy is, however, somewhat changed, since the expectation value of the pure exciton Hamiltonian is,

$$
\left\langle\psi\left|\hat{H}_{\mathrm{ex}}\right| \psi\right\rangle=\frac{\omega_{c}^{2}}{\left(\omega_{\mathrm{ex}}-\omega\right)^{2}+\omega_{c}^{2}}\left(E_{\mathrm{gap}}-E_{B}\right) .
$$

Similarly, any other pure exciton property will be modified according to (56) when the polariton wave function is taken into account.

It is seen from (55) that for $\left|\omega_{\mathrm{ex}}-\omega\right| \ll \omega_{c}, \psi$ is essentially the pure exciton wave function. Hence, the polariton is essentially an exciton in this important spectral range [which includes the range where exciton spectra are observed, typically a few $\omega_{\mathrm{LT}}$ around $\omega_{\mathrm{ex}}$, since $\left.\omega_{c} / \omega_{\mathrm{LT}} \simeq\left(\omega_{\mathrm{ex}} / 2 \omega_{\mathrm{LT}}\right)^{1 / 2} \gg 1\right]$. Although the polariton is described by the exciton wave function, it certainly has not a masslike dispersion curve. The $\omega-k$ relationship (see Fig. 1 ) is given by (53) or in exact form by (34) or (76), which is the dispersion curve for propagating light. Stated in a few words, the polariton for $\left|\omega_{\mathrm{ex}}-\omega\right| \ll \omega_{c}$ can be described as an exciton propagating through the solid with the speed of light of the solid. The interaction between light and matter is in the present case so strong that light and matter become mixed, and close to resonance the energy of the propagating "light" is mainly matterlike. This implies the possibility of constructing a modulator or deflector device where the matter properties of the polariton are affected by external fields to change the transmission characteristics and hence to modulate or deflect the beam. A discussion of such a device possibility will be published elsewhere.

Equation (55) is a one-quantum wave function. Hence, it is not possible to compute the polarization or electric field directly from (55) [e.g., by means of (12)]. A macroscopic field can be constructed by a superposition of many-quantum states (see, e.g., Glauber ${ }^{3}$ ). The operator for a macroscopic field with wave vector $\overrightarrow{\mathrm{k}}$ (in complex notation) is, in analogy to (42) or (44), given by

$$
\hat{F}=F_{1} \beta_{k} e^{i \overrightarrow{\mathrm{k}} \cdot \overrightarrow{\mathrm{r}}},
$$

where $\beta_{k}$ is the annihilation operator for the quanta of the field, and $F_{1}$ the "field per quantum." Introducing the creation operators $\alpha_{k}^{\dagger}, a_{k}^{\dagger}$, and $b_{k}^{\dagger}$ for polaritons, photons, and excitons, respectively, according to the definitions

$$
\psi=\alpha_{k}^{\dagger}|00\rangle, \quad \Phi_{1}=a_{k}^{\dagger}|00\rangle, \quad \Psi_{1}=b_{k}^{\dagger}|00\rangle,
$$

where $|00\rangle$ is the exciton-photon vacuum state, we can write (55) in terms of operators,

$\alpha_{k}=\frac{\omega_{\mathrm{ex}}-\omega}{\left[\left(\omega_{\mathrm{ex}}-\omega\right)^{2}+\omega_{c}^{2}\right]^{1 / 2}} a_{k}-i \frac{\omega_{c}}{\left[\left(\omega_{\mathrm{ex}}-\omega\right)^{2}+\omega_{c}^{2}\right]^{1 / 2}} b_{k}$.

From (58) we can, according to (57), construct the operator for the contribution to the electric field from a macroscopic polariton wave,

$$
E=E_{1} \frac{\omega_{\mathrm{ex}}-\omega}{\left[\left(\omega_{\mathrm{ex}}-\omega\right)^{2}+\omega_{c}^{2}\right]^{1 / 2}} a_{k} e^{i \overrightarrow{\mathrm{k}} \cdot \overrightarrow{\mathrm{r}}}
$$

where $E_{1}$ is the "electric field per pure photon" and is given by (45) (with $\omega_{p}$ replaced by $\omega$, since $\omega$ is the angular frequency of the wave). Similarly, the contribution to the polarization field from the polariton wave is given by 


$$
\hat{P}=P_{1} \frac{\omega_{c}}{\left[\left(\omega_{\mathrm{ex}}-\omega\right)^{2}+\omega_{c}^{2}\right]^{1 / 2}} b_{k} e^{i \overrightarrow{\mathrm{k}} \cdot \overrightarrow{\mathrm{r}}},
$$

where

$$
P_{1}=\frac{\mu}{V}=\left(\frac{\hbar \epsilon_{0} F}{2 \omega_{\mathrm{ex}} V}\right)^{1 / 2}
$$

is the "polarization per pure exciton" [compare with (33)]. Equations (14), (16), and (18) were used in (61).

According to (59) and (60) we can then introduce

$$
E_{0}=E_{1} \frac{\omega_{\mathrm{ex}}-\omega}{\left[\left(\omega_{\mathrm{ex}}-\omega\right)^{2}+\omega_{\mathrm{c}}^{2}\right]^{1 / 2}}
$$

and

$$
P_{0}=P_{1} \frac{\omega_{c}}{\left[\left(\omega_{\mathrm{ex}}-\omega\right)^{2}+\omega_{c}^{2}\right]^{1 / 2}}
$$

as the "electric field per polariton" (62) and as "the polarization per polariton" (63). Similar expressions for the polariton fields have previously been derived by Balslev ${ }^{4}$ from a macroscopic approach. $E_{0}$ and $P_{0}$ are the fields of the macroscopic state divided by the average number $\langle n\rangle$ of polaritons in that state. Hence

$$
\frac{P}{E}=\frac{\langle n\rangle P_{0}}{\langle n\rangle E_{0}}=\frac{P_{1}}{E_{1}} \frac{\omega_{c}}{\omega_{\mathrm{ex}}-\omega}=\epsilon_{0} \frac{F}{2 \omega\left(\omega_{\mathrm{ex}}-\omega\right)}
$$

using (30), (45), and (61). Since $P / E=\epsilon_{0}\left(\epsilon-\epsilon^{\prime}\right.$ ) we obtain

$$
\epsilon=\epsilon^{\prime}+\frac{F}{2 \omega\left(\omega_{\mathrm{ex}}-\omega\right)} \simeq \epsilon^{\prime}+\frac{F}{\omega_{\mathrm{ex}}^{2}-\omega^{2}} .
$$

Hence we have derived the dielectric constant (or dielectric response) from the microscopic approach by means of (39).

Finally, we shall consider the effect of introducing damping into the polariton fields (62) and (63). The damping occurs due to the scattering of the excitonic part of the polariton. Hence damping can be introduced by rewriting (49) as

$$
\frac{d a_{2}}{d t}=-\omega_{c} a_{1} e^{i\left(\omega_{\mathrm{ex}}-\omega_{p}\right) t}-\frac{1}{2} \Gamma a_{2} .
$$

Then if $\omega_{c}=0$ (no interaction), $\left|a_{2}\right|^{2}=\exp (-\Gamma t)$, i.e., the excitons decay with the rate $\Gamma$ which is then the phenomenological damping constant. The solution of (48) and (66) follows that of (48) and (49). The result is that $\omega_{\mathrm{ex}}$ everywhere must be exchanged by $\omega_{\mathrm{ex}}-i \Gamma / 2$. Hence the damping is easily introduced in the previous expressions by carrying out the replacement

$$
\omega_{\mathrm{ex}} \rightarrow \omega_{\mathrm{ex}}-\frac{1}{2} i \Gamma .
$$

Doing this in (65) we obtain the usual expression relevant for a damped harmonic oscillator. It is seen that the spectral structure is essentially damped away for

$$
\Gamma \gg \omega_{\mathrm{LT}} \text {. }
$$

Introducing (67) into (55), however, shows that the polariton wave function is relatively independent of damping for

$$
\Gamma \ll \omega_{c} .
$$

Thus, we have reached the important conclusion that although the exciton spectra are smeared out because of (68), the exciton is still the dominating part of the polariton (for $\left|\omega_{\mathrm{ex}}-\omega\right|<\omega_{c}$ ) when (69) is valid. Only when $\Gamma \gg \omega_{c}$ does the excitonic contribution in the polariton vanish.

Inserting damping into (62) and (63) by means of (67) results in the following polariton fields:

$$
\begin{aligned}
& E_{0}=E_{1} \frac{\omega_{\mathrm{ex}}-\omega-\frac{1}{2} i \Gamma}{\left[\left(\omega_{\mathrm{ex}}-\omega\right)^{2}+\omega_{c}^{2}+\Gamma^{2} / 4\right]^{1 / 2}}, \\
& P_{0}=P_{1} \frac{\omega_{c}}{\left[\left(\omega_{\mathrm{ex}}-\omega\right)^{2}+\omega_{c}^{2}+\Gamma^{2} / 4\right]^{1 / 2}} .
\end{aligned}
$$

\section{COMPARISON WITH EXACT POLARITON MODEL}

Finally, we shall compare the exact polariton solution derived by Hopfield ${ }^{1}$ from macroscopic considerations. The Hamiltonian of Hopfield is

$$
\begin{aligned}
\hat{H}=\sum_{k} & {\left[\hbar \omega_{p}\left(a_{k}^{\dagger} a_{k}+\frac{1}{2}\right)+\hbar \omega_{\mathrm{ex}}\left(b_{k}^{\dagger} b_{k}+\frac{1}{2}\right)\right.} \\
& +i \alpha\left(a_{k}^{\dagger} b_{k}-a_{k} b_{k}^{\dagger}+a_{-k} b_{k}-a_{-k}^{\dagger} b_{k}^{\dagger}\right) \\
& \left.+\beta\left(a_{k} a_{k}^{\dagger}+a_{k}^{\dagger} a_{k}+a_{k}^{\dagger} a_{-k}^{\dagger}+a_{-k} a_{k}\right)\right]
\end{aligned}
$$

where

$$
\alpha=\hbar \omega_{c}\left(\frac{\omega_{\mathrm{ex}}}{\omega_{p}}\right)^{1 / 2}
$$

and

$$
\beta=\frac{1}{2} \hbar \omega_{\mathrm{LT}}\left(\frac{\omega_{\mathrm{ex}}}{\omega_{p}}\right),
$$

and $a_{k}$ and $b_{k}$ are annihilation operators of photons and excitons, respectively, with wave vector $\overrightarrow{\mathbf{k}}$. Comparing (72) with (39) we see that the terms with $-k$ are dropped in (39) and the last term with $\beta$ is missing in (39). Furthermore, second quantization was not used for the excitons in (39) since the internal structure and the wave functions of the excitons were known in advance. The omission of the $-k$ terms corresponds, essentially, to dropping nonresonant terms such as $\omega_{p}+\omega_{\mathrm{ex}}$. This approximation is not serious and is often used close to resonance. However, as shown below [Eq. (83)] this approximation implies a considerably simpler polariton wave function. The neglect of the last term in (72) is more serious, since 
it leads to incorrect eigenenergies close to $k=0$. This term is due to the energy caused by the presence of the exciton dipole field in the background dielectric material (with dielectric constant $\left.\epsilon^{\prime}\right)$. This amount of energy is

$$
\frac{P_{1}^{2}}{\epsilon_{0} \epsilon^{\prime}} V=\epsilon_{0} \epsilon^{\prime} E^{\prime 2} V=\frac{\epsilon_{0} F}{\omega_{\mathrm{ex}}^{2}} E_{1}^{2} V=\hbar \omega_{\mathrm{LT}},
$$

where $E_{1}$ and $P_{1}$ are the "fields per quantum" given in (45) and (61) and $E^{\prime}$ is the magnitude of the electric field induced by the dipole field $P_{1}$. The inclusion of this term shifts the exciton energy at $k=0$ the amount $\hbar \omega_{\mathrm{LT}}$. The eigenenergies obtained from (72) are

$$
\hbar \omega=\frac{1}{2} \hbar\left\{\omega_{p}^{2}+\omega_{L}^{2} \pm\left[\left(\omega_{L}^{2}-\omega_{p}^{2}\right)^{2}+16 \omega_{p}^{2} \omega_{c}^{2}\right]^{1 / 2}\right\}^{1 / 2},
$$

where

$$
\omega_{\mathrm{L}}=\left(\omega_{\mathrm{ex}}^{2}+2 \omega_{\mathrm{ex}} \omega_{\mathrm{LT}}\right)^{1 / 2}
$$

is the longitudinal resonance frequency which is obtained for $k=0$. Comparison between (76) and (53) obtained from the simplified polariton model is shown in Fig. 1 for the $C$ exciton in $\mathrm{ZnO}$. $\mathrm{ZnO}$ has a strong exciton-photon interaction with $\hbar \omega_{c}$ $=122 \mathrm{meV}$. The parameters for $\mathrm{ZnO}$ are taken from Jensen. ${ }^{5}$ It is seen in Fig. 1 that the lower branch is relatively well described by (53). The upper branch computed from (53), however, starts at $\omega_{\mathrm{ex}}+\frac{1}{2} \omega_{\mathrm{LT}}$ for $k=0$ instead of $\omega_{\mathrm{ex}}+\omega_{\mathrm{LT}}$. This results in a relatively large discrepancy between (53) and (76) for $k \simeq 0$.

The exact polariton wave functions are created from the polariton creation operator ${ }^{1}$

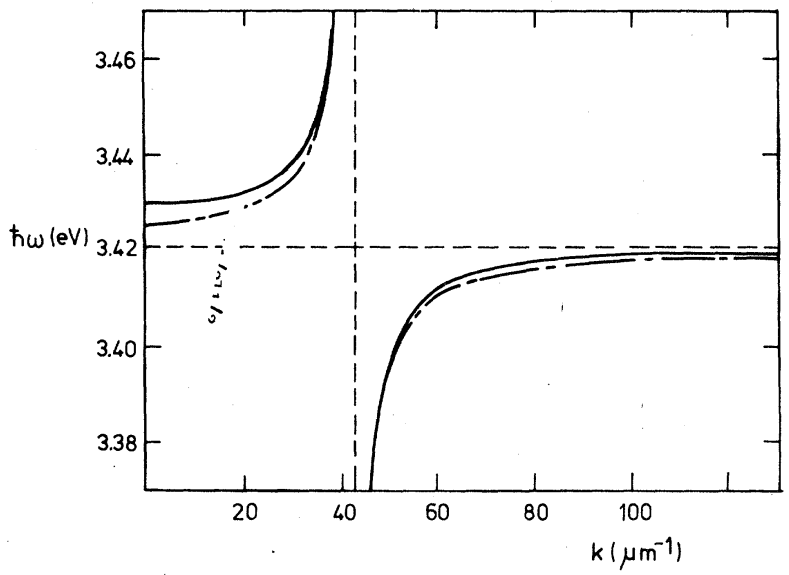

FIG. 1. Dispersion curves for the $C$ exciton in $\mathrm{ZnO}$. Dashed curves: Eigenenergies of pure photon and pure exciton states. Dot-dashed curves: Eigenenergies of polariton states according to (53). Solid curves: Exact eigenenergies of polaritons according to (76). The parameters $\hbar \omega_{\mathrm{ex}}=3.4210 \mathrm{eV}, F=0.37 \mathrm{eV}, \epsilon^{\prime}=6.2$ $\left(\hbar \omega_{\mathrm{LT}}=8.72 \mathrm{meV}\right)$ are taken from Ref. 5 .

$$
\begin{aligned}
\alpha_{k}^{\dagger}= & c_{11}^{*} a_{k}^{\dagger}+c_{12}^{*} b_{k}^{\dagger}+c_{13}^{*} a_{-k}+c_{14}^{*} b_{-k} \\
= & \left(c_{11}^{*}-c_{13}^{*}\right) a_{k}^{\dagger}+\left(c_{12}^{*}-c_{14}^{*}\right) b_{k}^{\dagger} \\
& +c_{13}^{*}\left(a_{-k}+a_{k}^{\dagger}\right)+c_{14}^{*}\left(b_{-k}+b_{k}^{\dagger}\right) .
\end{aligned}
$$

The last two terms of (78) represent a standing electromagnetic field and a standing polarization field, while the two first terms represent the propagating fields. The $c$ coefficients are given in Ref. 1,

$$
\begin{aligned}
& c_{11}^{*}-c_{13}^{*}=\frac{\omega_{\mathrm{ex}}^{2}-\omega^{2}}{\left[\left(\omega_{\mathrm{ex}}^{2}-\omega^{2}\right)^{2}+4 \omega_{c}^{2} \omega_{\mathrm{ex}}^{2}\right]^{1 / 2}}\left(\frac{\omega_{p}}{\omega}\right)^{1 / 2}, \\
& c_{12}^{*}-c_{14}^{*}=i \frac{2 \omega_{c} \omega}{\left[\left(\omega_{\mathrm{ex}}^{2}-\omega^{2}\right)^{2}+4 \omega_{c}^{2} \omega_{\mathrm{ex}}^{2}\right]^{1 / 2}}\left(\frac{\omega_{\mathrm{ex}}}{\omega}\right)^{1 / 2}, \\
& c_{13}^{*}=\left(c_{11}^{*}-c_{13}^{*}\right) \frac{\omega_{p}-\omega}{2 \omega_{p}}, \\
& c_{14}^{*}=i\left(c_{12}^{*}-c_{14}^{*}\right) \frac{\omega_{\mathrm{ex}}-\omega}{2 \omega} .
\end{aligned}
$$

Hence, the wave function derived in (55) for the dipole-active exciton (polariton) is only approximate. The more exact wave function can be deduced from the form of (78) to be

$$
\begin{aligned}
\psi=\frac{1}{N} & {\left[\left(\omega_{\mathrm{ex}}^{2}-\omega^{2}\right)\left(\frac{\omega_{p}}{\omega}\right)^{1 / 2} \Psi_{0} \Phi_{1}+2 i \omega_{c} \omega\left(\frac{\underline{\omega}_{\mathrm{ex}}}{\omega}\right)^{1 / 2} \Psi_{1} \Phi_{0}\right.} \\
& +\frac{\left(\omega_{\mathrm{ex}}^{2}-\omega^{2}\right)\left(\omega_{p}-\omega\right)}{2\left(\omega_{p} \omega\right)^{1 / 2}} \Psi_{0}\left(\Phi_{1-k}^{*}+\Phi_{1 k}\right) \\
& \left.+\frac{i \omega_{c} \omega_{\mathrm{ex}}\left(\omega_{\mathrm{ex}}-\omega\right)}{\left(\omega_{\mathrm{ex}} \omega\right)^{1 / 2}} \Phi_{0}\left(\Psi_{1-k}^{*}+\Psi_{1 k}\right)\right]
\end{aligned}
$$

where

$$
N=\left[\left(\omega_{\mathrm{ex}}^{2}-\omega^{2}\right)^{2}+4 \omega_{c}^{2} \omega_{\mathrm{ex}}^{2}\right]^{1 / 2} .
$$

Equation (83) is considerably more complicated than (55) obtained from the simplified treatment. When the nonresonant terms like $\omega_{p}+\omega$ are taken into account it is necessary to incorporate negative energies, too. This implies that standing waves appear in (83).

In analogy with (58), (59), and (60) we can from (65) and (83) construct the macroscopic field operators resulting in the following "fields per polariton":

$$
\begin{aligned}
& E_{0}=E_{1} \frac{\omega_{\mathrm{ex}}^{2}-\omega^{2}}{\left[\left(\omega_{\mathrm{ex}}^{2}-\omega^{2}\right)^{2}+4 \omega_{c}^{2} \omega_{\mathrm{ex}}^{2}\right]^{1 / 2}}, \\
& P_{0}=P_{1} \frac{2 \omega_{c} \omega}{\left[\left(\omega_{\mathrm{ex}}^{2}-\omega^{2}\right)^{2}+4 \omega_{c}^{2} \omega_{\mathrm{ex}}^{2}\right]^{1 / 2}}, \\
& E_{s}=E_{0} \frac{\omega_{p}-\omega}{2 \omega_{p}} \\
& P_{s}=P_{0} \frac{\omega_{\mathrm{ex}}-\omega}{2 \omega},
\end{aligned}
$$

where $E_{0}$ and $P_{0}$ are the propagating electric and 


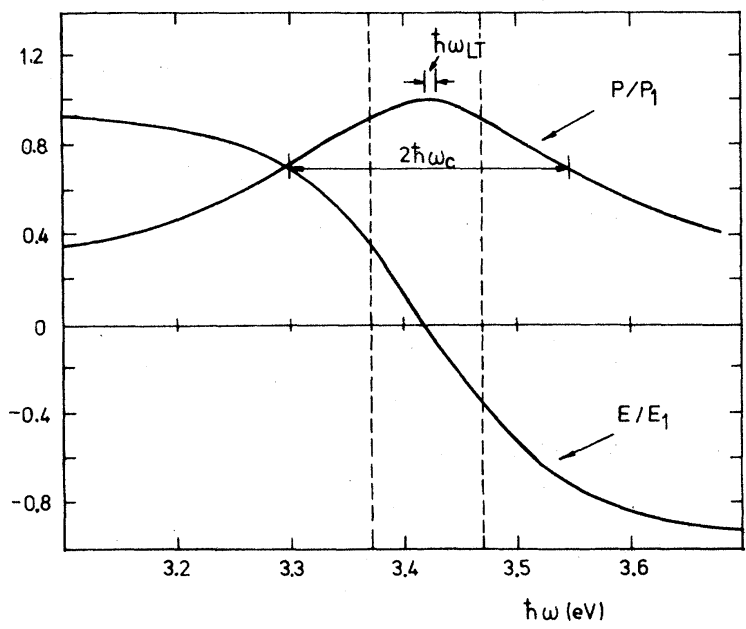

FIG. 2. Polariton fields for the $C$ exciton in $\mathrm{ZnO}$ computed from (85) and (86). The parameters are taken from Ref. 5. The exciton-photon interaction energy is. $\hbar \omega_{c}=122 \mathrm{meV}$. The spectral range used in Fig. 1 is indicated and the width of $\hbar \omega_{L T}$ is shown.

polarization fields, respectively, while $E_{s}$ and $P_{s}$ are the corresponding standing fields. $E_{1}$ and $P_{1}$ are given in $(45)$ and $(61)$. [The factors $\left(\omega_{p} / \omega\right)^{1 / 2}$ and $\left(\omega_{\mathrm{ex}} / \omega\right)^{1 / 2}$ in (83) are included in $E_{1}$ and $P_{1}$ in (85) and (86). Hence $E_{1}$ and $P_{1}$ depend only on $\omega$ as they also must since the fields oscillate with the frequency $\omega$.

In Fig. 2 the spectral dependence of the polariton fields (85) and (86) is shown. The approximate expressions (62) and (63) coincide with the exact curves in Fig. 2. The differences are too small to be seen in the scale applied, and (62) and (63) are, therefore, sufficiently accurate.

In conclusion of this comparison between the simplified and the exact polariton model it can be stated that the simplified wave function (55) represents the exact one (83) sufficiently well since only the propagating terms are of any practical importance. The derivation of the polariton fields from (55) is also sufficiently accurate. The eigenenergies (53) in the simplified model are, however, too approximate and either the exact expression (76) or the condition for light propagation (34) must be used.

Applying (76) [or (34)] and the wave function (55) for the interacting exciton-photon complex not only provides us with the detailed microscopic wave function for the dipole-active exciton and its dispersion relation, but it also yields a simple and clear physical picture of the interaction processes between photons and excitons which can help in the interpretations of experiments involving polaritons.

Finally, this simplified microscopic polariton model is compared with the exact solutions obtained for the macroscopic polariton model by Hopfield. It is seen that standing photon and exciton waves must be included in an exact microscopic polariton model. However, it is concluded that for practical purposes, only the propagating waves are of importance and the simple microscopic polariton wave function derived in the present work is then sufficient.
${ }^{1}$ J. J. Hopfield, Phys. Rev. 112, 1555 (1958).

${ }^{2}$ R. S. Knox, Theory of Excitons (Academic, New York, 1965).

${ }^{3}$ R. J. Glauber, Phys. Rev. 130, 2529 (1963); 131, 2766
(1963).

${ }^{4}$ I. Balslev, Phys. Rev. B 20, 648 (1979).

${ }^{5} \mathrm{G}$. Hvedstrup Jensen, Phys. Status Solidi B 64 , K51 (1974). 